\title{
PARADENTAL CYST ASSOCIATED WITH AN UNERUPTED WISDOM TOOTH
}

W.C. Ngeow, R.B. Zain, W.L. Chai. Paradental cyst associated with an unerupted wisdom tooth. Annal Dent Univ Malaya 2000; 7: 43-45.

\section{ABSTRACT}

The paradental cyst is an odontogenic cyst occurring near to the cervical margin of the lateral aspect of a root as a consequence of an inflammatory process in a periodontal pocket. A distinct form of the paradental cyst occurs not infrequently on the buccal aspects of erupted mandibular third molar, where there is an associated history of pericoronitis. A search of the literature revealed that these cysts had all been reported in relation to partially, newly or fully erupted molars. This report presents one case in which the cyst was noticed to be associated with an unerupted third molar. The histological appearance of the cyst and the gross relationship to the cemento-enamel junction is similar to those typical of paradental cysts reported in the literature. However, the radiographic and clinical appearance of the tooth being unerupted do not tally with paradental cyst and yet exclude the diagnosis of dentigerous cyst. The diagnosis of dental follicular tissue was excluded based on the histopathological presentation. The authors suggested that this case may represent an early form of paradental cyst which in the past may have been dismissed as dental follicular residues and thus, the prevalence of paradental cyst may have been under reported in the literature.

Key words: paradental cyst, unerupted tooth, dental follicular tissue.

\section{INTRODUCTION}

Since 1970, certain odontogenic cysts of inflammatory origin have been described under the terms inflammatory collateral cyst $(1,2)$, inflammatory lateral periodontal cyst (3), paradental cyst (4) and mandibular infected buccal cyst (5). These cysts were usually found adjacent to the cervical margins of partially or fully erupted mandibular molars with vital pulp. Most often they occurred at the buccal and/or distal and rarely, mesial aspects of the tooth roots. They were believed to have arisen as a consequence of inflammatory processes within the periodontal pockets. Radiographically, they usually presented as well-defined radiolucencies superimposed on the roots of the affected teeth. The histological features were similar to that of a radicular cyst.

The first publication by the World Health Organization (WHO) in 1971 on the classification of odontogenic tumours did not mention the existence of such cyst as a separate entity (6). Radicular cyst was
Case Report

\author{
W.C. Ngeow ${ }^{1}$, R.B. Zain ${ }^{2}$ and W.L. Chai \\ ${ }^{\prime}$ Department of Oral and Maxillofacial Surgery, \\ ${ }^{2}$ Department of Oral Pathology, \\ Oral Medicine and Periodontology and \\ ${ }^{3}$ General Dental Practice Unit, \\ Faculty of Dentistry, \\ University of Malaya, \\ 50603 Kuala Lumpur, \\ Malaysia \\ Corresponding author - W.C. Ngeow
}

listed as the only inflammatory odontogenic cyst. However, reports over the past two decades spurred debates over the existence of such cyst as a separate entity. Eventually, in the second edition of the WHO's publication on the classification of odontogenic tumours (1991), such cysts were recognised as a separate entity (7). The nomenclature given to such cysts was the "Paradental (Inflammatory Collateral, Mandibular Infected Buccal) cyst".

The paradental cyst was defined by WHO in 1991 as a cyst occurring near to the cervical margin of the lateral aspect of a root as a consequence of an inflammatory process in a periodontal pocket (7). It was believed that this cyst arose from odontogenic epithelium in the superficial part of the periodontal ligament related to a vital tooth. The WHO publication in 1991 had also described a distinct form of the paradental cyst that occurs on the buccal and distal aspect of an erupted mandibular molars with an associated history of pericoronitis (7).

In this paper a case of an atypical odontogenic cyst is presented. The suitability of the cyst being considered as an early paradental cyst is discussed.

\section{CASE REPORT}

A 23-year-old Chinese female came to the Department of Oral and Maxillofacial Surgery with a complaint of pain on her right mandibular third molar for the past few days. The tooth was partially erupted i.e. part of the crown could be seen clinically.

A dentopantomogram (DPT) taken showed that her right mandibular third molar was slightly mesially inclined. The DPT also showed the presence of an unerupted left mandibular third molar with a slight radiolucency distal to the tooth root area (Figure 1). Two overerupted left and right maxillary third molars were also present. The right maxillary and mandibular third molars were removed with the patient under local anaesthesia. 


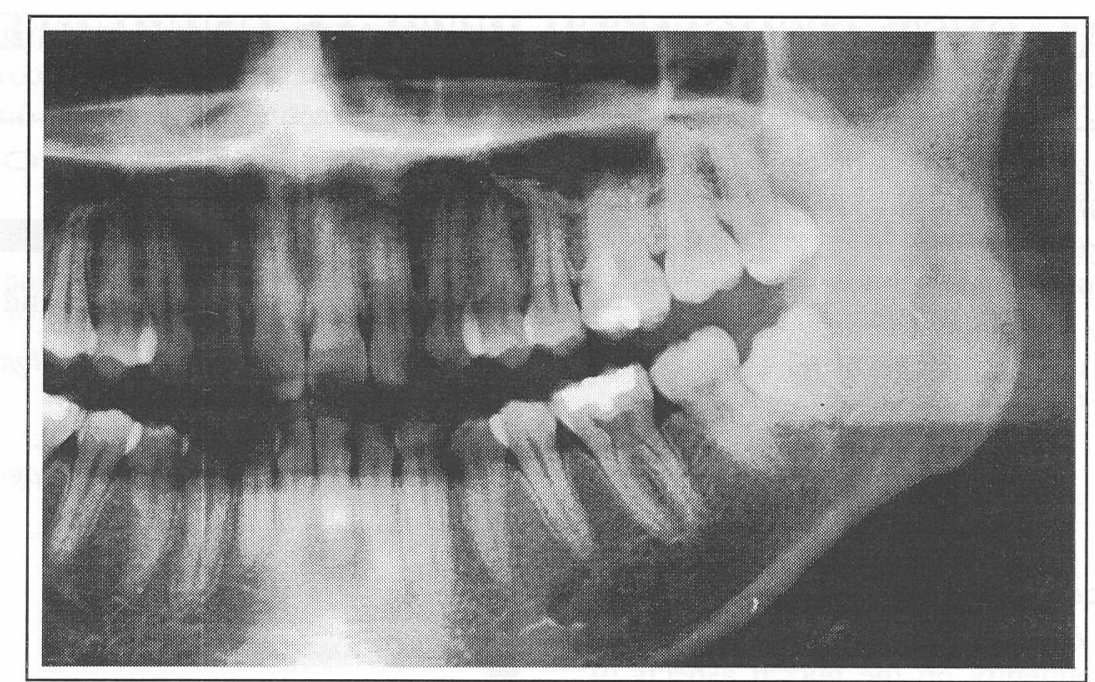

Figure 1: Radiographic picture showing the relationship of the unerupted left mandibular third molar to its adjacent structures.

Note the slight radiolucency distal to the left mandibular third molar root.

She returned two and a-half months later complaining of pain in the unerupted left mandibular third molar region. The left maxillary third molar was overerupted and was occluding onto the mucosal coverage of the unerupted left mandibular third molar. The overlying mucosa was tender to palpation. Cuspal marks and an erythematous area could be seen at the affected site.

An incision was made on the mucosal coverage of the left mandibular third molar and the tooth was elevated out. Upon doing so, a cystic lesion was found attached to the cemento-enamel junction of the distobuccal surface of the tooth. It was sent for histopathological examination. Healing was uneventful.

Histologically, the cystic specimen consisted of a lumen lined with non-keratinized stratified squamous epithelium. Focal aggregrates of chronic inflammatory cells were observed in the adjacent fibrous connective tissue cyst wall (Figure 2). The diagnosis was consistent with paradental cyst.

\section{DISCUSSION}

A search of the literature on paradental cyst or mandibular infected buccal cysts indicates no report of similar lesions being associated with an unerupted third molar. This case report is believed to be the first communication on such abnormality affecting an unerupted third molar.

The cyst in this case was located disto-buccally to the unerupted mandibular third molar. A slight radiolucency could be seen at the distal aspect of the tooth root area and was not enclosing the crown of the tooth. Upon surgery, the cyst-like tissue was attached to the cemento-enamel junction. This appearance is similar to those reported by Craig (4), Ackermann et al (8) and Fowler and Bowler (9). The diagnosis of

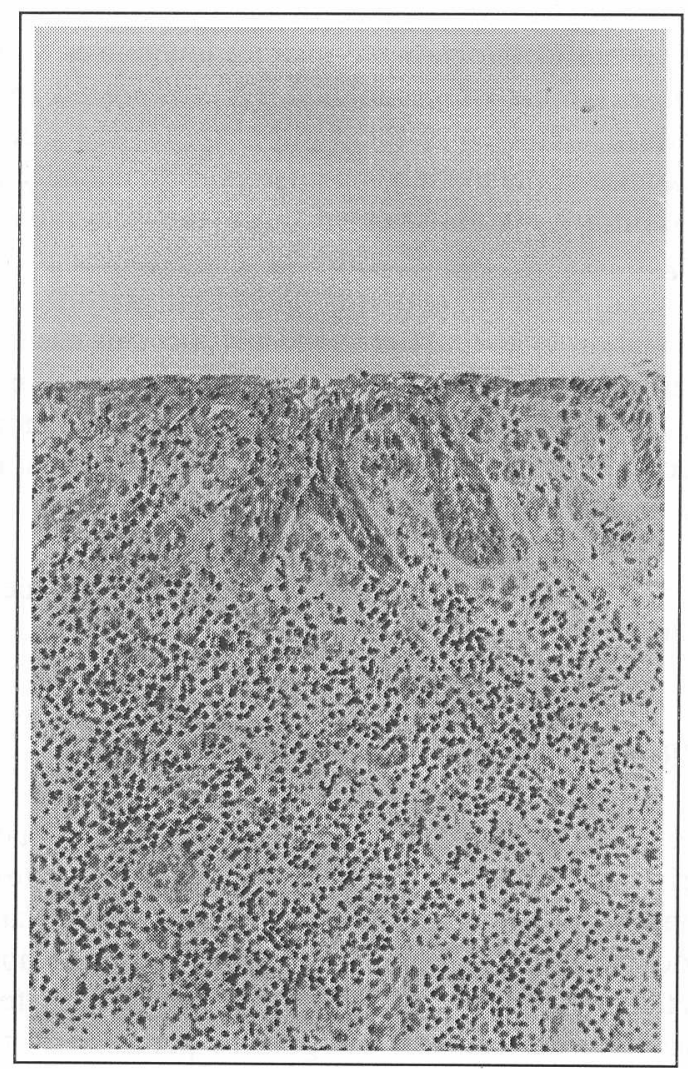

Figure 2: Photomicrograph showing a cystic lesion lined by a non-keratinized stratified squamous epithelium and the presence of chronic inflammatory cells in the cyst wall. (Original magnification - 50X)

dentigerous cyst was excluded in this case based on the radiographic appearance and the appearance during surgery where the cyst-like tissue did not surround the tooth crown. The radiographic appearance of the cyst was, however, not representative of a paradental cyst. This may be due to the fact that the overlapping of the 
cyst by the crown of the left mandibular third molar masking the radiolucency. The histolopathologic features of this cyst were similar to that reported by other authors and the WHO publication in 1991 (1-10).

This case differed from other reported cases and the WHO report in 1991 (7) because the left mandibular third molar was unerupted and gave no history of pericoronitis. The left maxillary third molar was however, occluding on the mucosa covering of the left mandibular third molar and such traumatic occlusion may be a source of subacute inflammation. However, there may be a possibility of infection via the distal pocket of the left mandibular second molar. The fissure on the occlusal surface of the extracted unerupted left mandibular third molar was darkly stained. This may indicate the presence of a periodontal pocket at the distal root of the left mandibular second molar adjacent to the unerupted left mandibular third molar. Such periodontal pocket would allow food debris, saliva and microorganism into the follicular space of the unerupted left mandibular third molar. The presence of the food debris, saliva and microorganism may cause staining on the occlusal fissures of the tooth. However, this periodontal pocket was not clinically detected, as the author (NWC) did not measure pocket depths around the left mandibular second molar prior to surgery.

All paradental cysts reported or mentioned in the literature were diagnosed shortly after tooth eruption. No suggestion was made to speculate when the cyst starts forming. The authors believe that the cyst had formed long before tooth eruption, as a consequence to inflammation.

The cyst in this case was an incidental finding during the removal of the unerupted left mandibular third molar. This type of cyst may not have been extensively reported because it is usually removed in the past together with the removal of the impacted or unerupted third molars. It may also have been thought to be a part of the dental follicular residues of the teeth (10). The diagnosis of follicular tissue in this case was excluded based on the histopathological appearance of the tissue attached to the cemento-enamel junction. Thus, the present case may be an early paradental cyst which in the past may have been dismissed as follicular residues and therefore, the prevalence of paradental cyst may have been under reported in the literature.

\section{REFERENCES}

1. Main DMG. Epithelial jaw cysts: a clinicopathological reappraisal. Br J Oral Surg 1970; 8: 114-25.

2. Main DMG. The enlargement of epithelial cysts. Odontol Rev 1970; 21: 29-49.

3. Main DMG. Epithelial jaw cysts: 10 years of the WHO classification. J Oral Pathol 1985; 14:1-7.

4. Craig GT. The paradental cyst: a specific inflammatory odontogenic cyst. Br. Dent J 1976; 141: 9-14.

5. Stoneman DW, Worth HM. The mandibular infected buccal cyst-molar area. Dental Radiography and Photography 1983; 56: 1-14.

6. Pindborg JJ, Kramer IRH, Torloni H. Histological typing of odontogenic tumours, jaw cysts and allied lesions. International Classification of Tumours. No. 5, Geneva: World Health Organisation, 1971; 44.

7. Kramer IRH, Pindborg JJ, Shear M. WHO Histological Typing of Odontogenic Tumours. 2nd ed. Berlin: Springer-Verlag, 1991; 42-5.

8. Ackermann G, Cohen MA, Altini M. The Paradental cyst. A clinicopathological study of 50 cases. Oral Surg Oral Med Oral Pathol 1987; 64: 308-312.

9. Fowler CB, Brannon RB. The paradental cyst. A clinicopathological study of six new cases and review of the literature. J Oral Maxillofac Surg 1989; 47: 243

10. Vedtofte P, Praetorius F. The Inflammatory paradental cyst. Oral Surg Oral Med Oral Pathol 1989; 68: 182-8. 